\title{
Semicontinuity of dimension and measure for locally scaling fractals
}

\author{
by
}

\author{
L. B. Jonker (Kingston, ON) and J. J. P. Veerman (Portland, OR)
}

\begin{abstract}
The basic question of this paper is: If you consider two iterated function systems close to each other in an appropriate topology, are the dimensions of their respective invariant sets close to each other? It is well known that the Hausdorff dimension (and Lebesgue measure) of the invariant set does not depend continuously on the iterated function system. Our main result is that (with a restriction on the "non-conformality" of the transformations) the Hausdorff dimension is a lower semicontinuous function in the $C^{1}$-topology of the transformations of the iterated function system. The same question is raised of the Lebesgue measure of the invariant set. Here we show that it is an upper semicontinuous function of the transformations. We also include some corollaries of these results, such as the equality of box and Hausdorff dimensions in these cases.
\end{abstract}

1. Introduction. In mathematics, as well as in the sciences, iterated function systems are commonly encountered and the dimensions of the associated invariant sets are considered to be important characteristics. The question we are ultimately interested in is: Suppose one has two slightly differing variants of an iterated function system (different digitizations, say, of the same underlying physical process). Is it likely that we will find two very different dimensions?

The answer we establish below is that the Hausdorff dimension is a semicontinuous function of the iterated function system, provided that the systems satisfy a weak conformality condition. This was known in some conformal cases (see [1]). An important corollary is that the Hausdorff dimension of the invariant set equals the so-called box dimension, which is much easier to calculate. Again this was observed in special cases ([3], [1]). The question that remains open is how scarce these discontinuities really are. In special cases, there are some results ([29], [10], [23]). We will come back to this at the end of the introduction.

2000 Mathematics Subject Classification: Primary 28A80; Secondary 28A78.

A preprint of an early version of this paper appeared as [28] in January, 1997. 
Let $H(\bar{I})$ be the space of compact subsets of the closed unit ball $\bar{I}$ in $\mathbb{R}^{n}$ and equipped with the Hausdorff metric. An iterated function system is a family of contracting mappings on $\mathbb{R}^{n}$ that map $\bar{I}$ into $I$. This induces a map from $H(\bar{I})$ to itself. In the standard examples, including those given at the end of this introduction, these families of mappings are finite. For the purposes of the theorems they only need to form a compact set in a suitable topology. The appropriate topologies will be introduced later on.

Definition 1.1. Let $\mathcal{F}$ be a collection of contracting homeomorphisms on $\mathbb{R}^{n}$. The associated iterated function system is the map $\phi_{\mathcal{F}}: H(\bar{I}) \rightarrow$ $H(\bar{I})$, determined by

$$
\phi_{\mathcal{F}}(A)=\bigcup_{f \in \mathcal{F}} f(A)
$$

The fact that such iterated function systems are contractions, provided $\mathcal{F}$ is a finite set, was observed in [8]. Then, if $A \in H(\bar{I})$, it follows that $\left\{\phi_{\mathcal{F}}^{n}(A)\right\}_{n}$ converges uniformly to a uniquely defined compact set $\Lambda(\mathcal{F})$ (the fixed point). Furthermore, $\Lambda(\mathcal{F})$ is invariant under $\phi_{\mathcal{F}}$ and is given by the intersection

$$
\Lambda(\mathcal{F})=\bigcap_{n>0} \phi_{\mathcal{F}}^{n}(\bar{I})
$$

By definition, then, this fixed point is a compact set for which we have

$$
\phi_{\mathcal{F}}(\Lambda)=\Lambda \text {. }
$$

For completeness, we include the definitions of the Hausdorff dimension and the limit capacity of a set. A thorough treatment of these topics is found in [4].

Definition 1.2. Let $\mathcal{V}_{\delta}$ be the collection of covers of a set $C$ whose members have diameter less than or equal to $\delta$, and let

$$
\mathcal{H}_{\delta}^{d}(C)=\inf _{\mathcal{V} \in \mathcal{V}_{\delta}} \sum_{V_{i} \in \mathcal{V}}\left|V_{i}\right|^{d} .
$$

The Hausdorff dimension $\operatorname{Hdim}(C)$ of $C$ is given by

$$
\operatorname{Hdim}(C)=\inf \left\{d: \lim _{\delta \rightarrow 0} \mathcal{H}_{\delta}^{d}(C)=0\right\} .
$$

Definition 1.3. Let $C$ be a compact set in $\mathbb{R}^{n}$. The limit capacity (also known as the upper box counting dimension $) d_{\mathrm{c}}(C)$ of $C$ is given by

$$
d_{\mathrm{c}}(C)=\limsup _{\delta \rightarrow 0} \frac{\ln \nu_{C}(\delta)}{-\ln \delta},
$$

where $\nu_{C}(\delta)$ is the minimum number of balls of radius $\delta$ needed to cover $\mathrm{C}$.

In order to formulate the main results of the paper, we define spaces $\mathbb{G}^{0}$ and $\mathbb{K}^{1}$ of contractions on $\bar{I}$ and put topologies on them. 
The space $\mathbb{G}^{0}$ is intended for a study of the Lebesgue measure of $\Lambda$, and is given a topology induced by the $C^{0}$-metric on the space of continuous mappings.

For the distortion estimates in Section 4 and the dimension estimates in Section 5 , it is vitally important to have control over the derivatives. Thus to be able to work with the dimension, we introduce another space. $\mathbb{K}^{1}$ is a subspace consisting of contracting diffeomorphisms with uniformly Hölder continuous derivatives.

In each case $\left(\mathbb{G}^{0}\right.$ and $\left.\mathbb{K}^{1}\right)$, the metric induces a Hausdorff metric on the space of compact sets of contracting functions. These metric spaces, whose elements are compact subsets of $\mathbb{G}^{0}$ and $\mathbb{K}^{1}$, will be denoted by $H\left(\mathbb{G}^{0}\right)$ and $H\left(\mathbb{K}^{1}\right)$ respectively. The purpose of Section 2 is to define all these notions carefully. It is easy to check, once the precise definitions have been presented, that if $\mathcal{F}$ is a point in one of these metric spaces, then $\phi_{\mathcal{F}}$ and $\Lambda(\mathcal{F})$ are well defined, just as in the case when $\mathcal{F}$ is a finite set. We define two functions:

Definition 1.4. Let $\mu: H\left(\mathbb{G}^{0}\right) \rightarrow \mathbb{R}^{+}$be the function that assigns the Lebesgue measure of $\Lambda(\mathcal{F})$ to $\mathcal{F}$.

Definition 1.5. Let Hdim $: H\left(\mathbb{K}^{1}\right) \rightarrow \mathbb{R}^{+}$be the function that assigns the Hausdorff dimension of $\Lambda(\mathcal{F})$ to $\mathcal{F}$.

In Section 3 we prove the following result.

TheOrem A. The function $\mu$ is upper semicontinuous on $H\left(\mathbb{G}^{0}\right)$.

As it happens, the theory involving derivatives is quite subtle. Our proofs require, in Section 5, the added assumption that the system is semiconformal.

Definition 1.6. A subset $\mathcal{F} \subset \mathbb{K}_{\alpha, C}^{1}$ is called semiconformal if

$$
\lim _{n \rightarrow \infty} \max _{f_{i} \in \mathcal{F}, x_{0} \in \bar{I}} \frac{1}{n} \ln \left\|D\left(f_{n} \ldots f_{1}\right)_{\mid x_{0}}\right\| \cdot\left\|\left(D\left(f_{n} \ldots f_{1}\right)_{\mid x_{0}}\right)^{-1}\right\|=0 .
$$

The subset $\mathbb{S} \subset H\left(\mathbb{K}^{1}\right)$ is the collection of semiconformal systems $\mathcal{F}$. When $\mathcal{F}$ is semiconformal, we also say that the induced contraction $\phi_{\mathcal{F}}$ is semiconformal.

Note that this is more general than conformal. For example, if the derivatives of each of the functions in $\mathcal{F}$ are constant and equal, then it can be seen without much difficulty that the assumption of semiconformality is equivalent to the assumption that the eigenvalues are equal in modulus.

The proof of the main theorem uses estimates of ratios of derivatives of long compositions taken at different points. Distortion calculations of this sort have been done before in the case of one-dimensional systems. In Section 4 , we do these calculations for arbitrary dimension. 
Definition 1.7. The subset $\mathbb{O} \subset H\left(\mathbb{K}^{1}\right)$ is the collection of systems $\mathcal{F}$ satisfying the strong separation condition. That is, $\mathcal{F}$ consists of finitely many functions $f_{i}$ and there is an open set $V$ containing $\Lambda(\mathcal{F})$ such that $f_{i}(V) \cap f_{j}(V)$ is empty whenever $i \neq j$.

In Section 5, we prove two results that imply the following:

Theorem B. The function $\mathrm{Hdim}: H\left(\mathbb{K}^{1}\right) \rightarrow \mathbb{R}$ is continuous on $\mathbb{S} \cap \mathbb{O}$ and lower semicontinuous on $\mathbb{S}$.

\section{COROllary C. On $\mathbb{S}$ the Hausdorff dimension and the limit capacity are equal.}

The equality to the limit capacity is important because the limit capacity lends itself to numerical calculations, and because it is the concept used in embedding theorems (see [21]). It should be pointed out that in [3], Falconer presents distortion estimates which could be used to show that conformal systems satisfy Theorem 4 in that paper and therefore give rise to attractors whose Hausdorff dimension and limit capacity are equal.

The principal result, however, is the semicontinuity for semiconformal systems. Earlier work on the semicontinuity of Hausdorff dimension was done for systems in $\mathbb{R}^{1}$, for example, in [18]. There it was shown for piecewise monotonic expanding maps of an interval into $\mathbb{R}$ that the Hausdorff dimension of the invariant set is lower semicontinuous in the $C^{1}$ topology.

The examples below will show that continuity does not hold under our hypotheses unless we include the strong separation condition. There are results in the literature that prove continuity in special circumstances that take the place of the strong separation condition. For example, in [14] and in [16] one finds proofs of the continuity of the Hausdorff dimensions of a hyperbolic invariant set and its intersections with stable and unstable manifolds, for diffeomorphisms on a compact 2-manifold without boundary. It is also shown in these papers that under certain hypotheses, the Hausdorff dimension and the limit capacity are equal. In [24], Takens obtains related results for hyperbolic diffeomorphisms in dimension 2. In some cases smoothness of the dimension can also be proved. For example, in [19] it is shown that the Hausdorff dimension of the Julia set of a rational function depends analytically on the function (if the function is expanding on its Julia set). Other researchers have related the Hausdorff dimension to other quantities, such as the Lyapunov exponents [30].

Here are some examples to illustrate the subtlety of the problem we are studying. Note that they establish that the functions mentioned are not continuous. 
For $t \in[0,1 / 2]$, let $\mathcal{F}_{t}$ be given by $\left\{f_{i}\right\}_{i=0}^{2}$ where

$$
f_{0}(x)=\frac{x}{3}, \quad f_{1}(x)=\frac{x+t}{3}, \quad f_{2}(x)=\frac{x+1}{3} .
$$

Note that each function maps the unit interval into itself, thus the system is a contraction of $H([0,1])$ into itself. The dimension and measure of the invariant set now depend only on the parameter $t$.

TheOREM 1.8. Let $\mathcal{F}_{t}$ be the system just described. Then:

(i) If $t=p / q$ is rational and $p q \equiv 2(\bmod 3)$ then $\mu(t)=1 / q$.

(ii) If $t=p / q$ is rational and $p q \not \equiv 2(\bmod 3)$ then $\operatorname{Hdim}(t)<1$.

(iii) For all irrational $t, \mu(t)=0$.

(iv) For almost all $t, \operatorname{Hdim}(t)=1$.

The condition that $p q \equiv 2(\bmod 3)$ is equivalent to the condition that $\{0, p, q\}$ is a complete set of representatives of the coset space $\mathbb{Z} / 3 \mathbb{Z}$. The first result in Theorem 1.8 is a special case of a result proved in [7], with a more geometrical proof in [25]. The second statement is implied by Theorem 4.1 of [11] and Theorem 2.3 of [26]. The third part of the theorem is due to [12]. The last part follows from a general theorem by Marstrand ([13]). These results are also described in [10].

It is clear that in the system of this theorem the Hausdorff dimension is not continuous as a function of $t$. The question now arises how scarce these discontinuities are. Furstenberg conjectured that for this example the values of $t$ mentioned in part (ii) are the only discontinuities. That is, for all irrational $t, \operatorname{Hdim}(t)=1$. In [10] and independently in [29] it was proved that for certain Liouville numbers the Hausdorff dimension is 1 . In [23] it was proved that if $t$ is irrational, the Hausdorff dimension is always greater than 0.767 .

Another example is the following family of systems $\mathcal{G}_{\lambda}$ given by $\left\{f_{i}\right\}_{i=0}^{2}$ where

$$
f_{0}(x)=\lambda x, \quad f_{1}(x)=\lambda(x+1), \quad f_{2}(x)=\lambda(x+3) .
$$

Using techniques very different from ours, Pollicott and Simon [17], in answer to a question posed by Keane, have recently shown that for almost all $\lambda<$ $1 / 3$ the Hausdorff dimension $\operatorname{Hdim}\left(\mathcal{G}_{\lambda}\right)$ is equal to $-\ln 3 / \ln \lambda$, while there is a dense subset of $[1 / 4,1 / 3]$ such that if $\lambda$ belongs to this set then $\operatorname{Hdim}\left(\mathcal{G}_{\lambda}\right)$ is strictly less than $-\ln 3 / \ln \lambda$. This is related to a problem considered by Erdős: for which $\lambda \in[1 / 3,1]$ is the invariant density related to this system singular with respect to Lebesgue measure? (See [22] and references therein.)

Acknowledgments. We are grateful to Marcelo Viana for useful conversations on an early version of Theorem 5.6. 
2. Definitions. Let $\bar{I}$ be the closed unit ball in $\mathbb{R}^{n}$. The space $H(\bar{I})$ is the collection of compact subsets of $\bar{I}$. For any compact $A$, denote its $\varepsilon$-neighborhood in $\bar{I}$ by $N_{\varepsilon}(A)$. We topologize $H(\bar{I})$ by endowing it with the Hausdorff metric Hd: if $A_{1}, A_{2} \in H(\bar{I})$, then

$$
\operatorname{Hd}\left(A_{1}, A_{2}\right)=\max \left\{\varepsilon_{1}, \varepsilon_{2}\right\} \quad \text { where } \varepsilon_{i}=\inf \left\{\varepsilon: N_{\varepsilon}\left(A_{i}\right) \supset A_{j}, j \neq i\right\} .
$$

With this topology $H(\bar{I})$ becomes a complete, compact metric space (see [8]). More generally, if $X$ is any metric space we can use the same construction to define a Hausdorff metric on the space $H(X)$ of all compact subsets of $X$.

We now present precise definitions of the spaces $\mathbb{G}^{0}$ and $\mathbb{K}^{1}$ of contractions.

Definition 2.1. The space $\mathbb{G}^{0}$ is the set of all uniform contractions $f: \bar{I} \rightarrow I$ (for each $f \in \mathbb{G}^{0}$ there is a number $0<L<1$ such that for any $x, y \in \bar{I}$ we have $d(f(x), f(y)) \leq L d(x, y))$. $\mathbb{G}^{0}$ is a metric space with the metric

$$
d_{0}(f, g)=\sup \{|f(x)-g(x)|: x \in \bar{I}\} .
$$

We also let $\mathbb{G}_{L}^{0}$ denote the subset of $\mathbb{G}^{0}$ consisting of all contractions with Lipschitz coefficient $L$.

Lemma 2.2. If $\mathcal{F}$ is a compact subset of $\mathbb{G}_{L}^{0}$, that is, if $\mathcal{F} \in H\left(\mathbb{G}_{L}^{0}\right)$, then $\phi_{\mathcal{F}}$ is a contraction on $H(\bar{I})$. In particular, $\phi_{\mathcal{F}}$ is continuous.

Proof. The proof is easy, and is left to the reader.

When in Section 3 we prove the semicontinuity of the measure of $\Lambda(\mathcal{F})$ as a function of $\mathcal{F}$, the topology we will be using is that induced by the Hausdorff metric on $H\left(\mathbb{G}^{0}\right)$ and on its subspace $H\left(\mathbb{G}_{L}^{0}\right)$ :

$$
\begin{aligned}
\operatorname{Hd}_{0}(\mathcal{F}, \mathcal{G})=\inf \{\varepsilon: & \forall f \in \mathcal{F} \exists g \in \mathcal{G} \text { such that } d_{0}(f, g)<\varepsilon \text { and } \\
& \left.\forall g \in \mathcal{G} \exists f \in \mathcal{F} \text { such that } d_{0}(f, g)<\varepsilon\right\} .
\end{aligned}
$$

To work with the Hausdorff dimension we need to establish control of the derivatives of the elements of $\mathcal{F}$. This is done in the following definitions:

Definition 2.3. The space $\mathbb{K}^{1}$ is the set of uniformly contracting diffeomorphisms $f$ from $\bar{I}$ to $I$, with the $C^{1}$-metric

$$
d_{1}(f, g)=\sup _{x \in \bar{I}}\left(|f(x)-g(x)|+\left\|D f_{\mid x}-D g_{\mid x}\right\|\right) .
$$

Note that by definition $\mathbb{K}^{1}$ is a subset of $\mathbb{G}^{0}$. Once again, the estimates in Section 5 also require the uniform Hölder continuity of the derivative.

Definition 2.4. Let $\alpha$ and $C$ be positive constants. The space $\mathbb{K}_{\alpha, C}^{1}$ is the subset of $\mathbb{K}^{1}$ consisting of all $f$ satisfying

$$
\left\|D f_{\mid x}-D f_{\mid y}\right\| \leq C|x-y|^{\alpha} .
$$

$\mathbb{K}_{\alpha, C}^{1}$ is a metric subspace of $\mathbb{K}^{1}$. 
Note that this time, if $\mathcal{F}$ is a compact subset of $\mathbb{K}^{1}$, then there is necessarily a uniform contraction factor $0<L<1$ for $\mathcal{F}$, and this holds true even for a sufficiently small neighborhood of $\mathcal{F}$ in $\mathbb{K}^{1}$.

DEFINITION 2.5. A differentiable iterated function system is a map $\phi_{\mathcal{F}}$ : $H(\bar{I}) \rightarrow H(\bar{I})$ induced by a compact subset $\mathcal{F}$ of $\mathbb{K}^{1}$, that is, by $\mathcal{F} \in H\left(\mathbb{K}^{1}\right)$.

When in Section 5 we prove the semicontinuity of the Hausdorff dimension of $\Lambda(\mathcal{F})$ as a function of $\mathcal{F}$, the topology we will be using is that induced by the Hausdorff metric on $H\left(\mathbb{K}^{1}\right)$ and on its subspace $H\left(\mathbb{K}_{\alpha, C}^{1}\right)$. That is,

$$
\begin{array}{r}
\operatorname{Hd}_{1}(\mathcal{F}, \mathcal{G})=\inf \left\{\varepsilon: \forall f \in \mathcal{F} \exists g \in \mathcal{G} \text { such that } d_{1}(f, g)<\varepsilon\right. \text { and } \\
\left.\forall g \in \mathcal{G} \exists f \in \mathcal{F} \text { such that } d_{1}(f, g)<\varepsilon\right\} .
\end{array}
$$

3. The measure estimate. We will show that if $\mathcal{F} \in H\left(\mathbb{G}_{L}^{0}\right)$ then $\mathcal{F}$ is a point of upper semicontinuity of the function $\mu$. The modulus of semicontinuity is estimated in terms of the limit capacity of the boundary of the invariant set. As far as we are aware these results have not been published by other authors.

As is well known, the fixed point set is a continuous function of $\mathcal{F}$. More precisely:

Proposition 3.1. $\operatorname{Hd}(\Lambda(\mathcal{F}), \Lambda(\mathcal{G})) \leq \frac{\operatorname{Hd}_{0}(\mathcal{F}, \mathcal{G})}{1-L}$.

Proof. Observe that by the triangle inequality

$$
\begin{aligned}
\operatorname{Hd}(\Lambda(\mathcal{F}), \Lambda(\mathcal{G})) & =\operatorname{Hd}\left(\phi_{\mathcal{F}} \Lambda(\mathcal{F}), \phi_{\mathcal{G}} \Lambda(\mathcal{G})\right) \\
& \leq \operatorname{Hd}\left(\phi_{\mathcal{F}} \Lambda(\mathcal{F}), \phi_{\mathcal{F}} \Lambda(\mathcal{G})\right)+\operatorname{Hd}\left(\phi_{\mathcal{F}} \Lambda(\mathcal{G}), \phi_{\mathcal{G}} \Lambda(\mathcal{G})\right) \\
& \leq L \cdot \operatorname{Hd}(\Lambda(\mathcal{F}), \Lambda(\mathcal{G}))+\operatorname{Hd}_{0}(\mathcal{F}, \mathcal{G}) .
\end{aligned}
$$

TheOREM A. The function $\mu: H\left(\mathbb{G}_{L}^{0}\right) \rightarrow \mathbb{R}$ is upper semicontinuous.

Proof. The function $\mu$ is the composition of $\Lambda$ (which is continuous by Proposition 3.1) and the Lebesgue measure function on $H(\bar{I})$, which we will also denote by $\mu$ since no confusion is possible. It suffices to prove that the latter is semicontinuous.

Suppose $\Lambda_{0} \in H(\bar{I})$ and suppose $\varepsilon>0$ is given.

Clearly, the neighborhoods $N_{1 / n}\left(\Lambda_{0}\right)$ form a decreasing collection of sets with

$$
\lim _{n \rightarrow \infty} N_{1 / n}\left(\Lambda_{0}\right)=\Lambda_{0}
$$

Since $\mu$ is a continuous measure this implies that

$$
\lim _{n \rightarrow \infty} \mu\left(N_{1 / n}\left(\Lambda_{0}\right)\right)=\mu\left(\lim _{n \rightarrow \infty} N_{1 / n}\left(\Lambda_{0}\right)\right)=\mu\left(\Lambda_{0}\right) .
$$

Therefore, if $n$ is large enough, $\operatorname{Hd}\left(\Lambda, \Lambda_{0}\right)<1 / n$ implies $\mu(\Lambda)<\mu\left(\Lambda_{0}\right)+\varepsilon$. 
Notice that the semicontinuity is not uniform (it cannot be according to the examples in the introduction). But we can, in fact, estimate its modulus.

In the following, $\partial \Lambda$ denotes the boundary of the set $\Lambda$.

Proposition 3.2. Suppose $\Lambda_{0} \in H(\bar{I})$, and $d>d_{0}=d_{\mathrm{c}}\left(\partial \Lambda_{0}\right)$. Then for any $\varepsilon>0$ and sufficiently small $\Delta>0$ the following is true: If $\Lambda \in N_{\Delta}\left(\Lambda_{0}\right)$, then

$$
\mu(\Lambda) \leq \mu\left(\Lambda_{0}\right)+\varepsilon \Delta^{n-d} .
$$

Proof. Observe that $\partial \Lambda_{0}$ can be covered by $p(\Delta) \Delta^{-d_{0}}$ balls of radius $\Delta$, where by definition

$$
\limsup _{\Delta \rightarrow 0} \frac{\ln p(\Delta)}{-\ln \Delta}=0
$$

If we increase the radius of each of these balls to $3 \Delta$, keeping their centers fixed, then the larger balls together with $\Lambda_{0}$ will cover $N_{\Delta}\left(\Lambda_{0}\right)$. Thus, if $K_{n}$ is the volume of the unit ball in $\mathbb{R}^{n}$, then

$$
\begin{aligned}
\mu(\Lambda) & \leq \mu\left(\Lambda_{0}\right)+\mu\left(N_{\Delta}\left(\partial \Lambda_{0}\right)\right) \leq \mu\left(\Lambda_{0}\right)+(3 \Delta)^{n} K_{n} \cdot p(\Delta) \Delta^{-d_{0}} \\
& =\mu\left(\Lambda_{0}\right)+(3 \Delta)^{n} K_{n} \cdot p(\Delta) \Delta^{\delta} \cdot \Delta^{-d_{0}-\delta} .
\end{aligned}
$$

The term $p(\Delta) \Delta^{\delta}$ tends to zero. Hence, for $\delta=d-d_{0}$ and $\Delta$ sufficiently small, the product $3^{n} \cdot K_{n} \cdot p(\Delta) \Delta^{\delta}$ is less than $\varepsilon$.

Corollary 3.3. Fix $\mathcal{F}_{0} \in \mathbb{G}_{L}^{0}$. Then for $d>d_{\mathrm{c}}\left(\partial \Lambda\left(\mathcal{F}_{0}\right)\right)$, and for any $\varepsilon>0$ and sufficiently small $\Delta>0$, the following is true:

$$
\mathcal{F} \in \mathbb{G}_{L}^{0} \text { and } d\left(\mathcal{F}, \mathcal{F}_{0}\right) \leq \Delta \Rightarrow \mu(\Lambda(\mathcal{F})) \leq \mu\left(\Lambda\left(\mathcal{F}_{0}\right)\right)+\varepsilon \Delta^{n-d} .
$$

This relation is very useful in the context of Theorem 1.8: Fix $t_{0}$ to be an irrational number, and approximate it by rational numbers of the form $p / q$ with the property that $p q \equiv 2(\bmod 3)$ (it is easy to see that these rationals are dense). One sees that

$$
\mu(p / q) \leq \mu\left(t_{0}\right)+\varepsilon\left|t_{0}-p / q\right|^{1-d} .
$$

Since in this case the boundary of $\Lambda\left(\mathcal{F}_{0}\right)$ equals the entire set $\Lambda\left(\mathcal{F}_{0}\right)$ (because its measure is zero), and by Corollary $\mathrm{C}$, we may take $\operatorname{Hdim}\left(t_{0}\right)$ instead of $d_{\mathrm{c}}\left(\partial \Lambda\left(\mathcal{F}_{0}\right)\right)$. Again using Theorem 1.8 , we see that $\mu(p / q)=1 / q$ and that $\mu\left(t_{0}\right)=0$. Thus for any $\varepsilon>0$ and $p / q$ sufficiently close to $t_{0}$,

$$
d>\operatorname{Hdim}\left(t_{0}\right) \Rightarrow 1 / q \leq \varepsilon\left|t_{0}-p / q\right|^{1-d} .
$$

This is used in [29] to obtain estimates for the dimension for some irrational $t_{0}$. (Kenyon [10] obtained these estimates via a different method.)

4. The distortion estimate. In one dimension, there is an elegant theory to obtain distortion estimates. This theory is described in various research papers and expository works (for example [6], [15] and [27]). The first step in this line of thought is the following. Consider the forward orbit of an 
interval $I_{0}$ under a function $f$ and write $I_{i}=f\left(I_{i-1}\right)$. Let $|\ln | D f||_{\alpha}$ denote the $\alpha$-Hölder norm of the logarithm of the derivative $D f$ of $f$ (restricted to the forward orbit of $I_{0}$ ). The distortion is the ratio of derivatives of high iterates of $f$ on a small interval $I_{0}$. It is given by the following expression (see the references listed above):

$$
\sup _{x_{0}, y_{0} \in I_{0}}|\ln | \frac{D f^{n}\left(x_{0}\right)}{D f^{n}\left(y_{0}\right)}|| \leq|\ln | D f||_{\alpha} \cdot \sum_{i}\left|I_{i}\right|^{\alpha} .
$$

In this section we mimic the derivation of the above estimate for dimensions greater than one. Also we will not be iterating a single function, but rather a sequence of functions picked from a compact set fixed beforehand. The latter generalization complicates the notation, but not the mathematics.

There is a classical distortion theory for conformal maps. Distortion for non-conformal maps has also been investigated by various authors in several papers ([9], [5]). In these papers a condition is established that guarantees that the image of a sufficiently small ball $B$ under a composition $g$ of contractions satisfies: there are constants $c_{1}$ and $c_{2}$ such that

$$
c_{1} D g(B) \subset g(B) \subset c_{2} D g(B) .
$$

In our context we need something stronger: we need to establish that the image of a ball is not too far away from a ball (see Theorem 4.10).

Let us start by outlining the general idea of the estimates. Suppose $\mathcal{F}$ is a compact set of diffeomorphisms in $\mathbb{K}_{\alpha, C}^{1}$. Consider $B_{0}=\bar{I}$ and a sequence of contractions $f_{i} \in \mathcal{F}$. Define $f_{i}\left(B_{i-1}\right)=B_{i}$. We will express the distortion, or non-linearity, in terms of a sum of powers of the diameters of the regions $B_{i}$, just as in the one-dimensional case, except that now there will be a penalty for non-conformality. More precisely, choose two points $x_{0}$ and $y_{0}$ in $B_{0}$ and denote the images of $x_{i-1}$ and $y_{i-1}$ under $f_{i}$ by $x_{i}$ and $y_{i}$. The usual operator norm is written as $\|\cdot\|$. To construct the higher dimensional analogue of the left hand side of (4.1) we define

$$
C_{n}=C_{n}\left(f_{n} \ldots f_{1} ; x_{0}, y_{0}\right)=\left(D\left(f_{n} \ldots f_{1}\right)_{\mid x_{0}}\right)^{-1} \cdot D\left(f_{n} \ldots f_{1}\right)_{\mid y_{0}} .
$$

The idea is to obtain estimates for the logarithm of $\sup _{x_{0}, y_{0} \in \bar{I}, f_{j} \in \mathcal{F}}\left\|C_{n}\right\|$.

Definition 4.1. For $\mathcal{F} \in \mathbb{K}^{1}$, we define the distortion as

$$
D(n)=\max _{i \leq n} \sup _{x, y \in \bar{I}, f_{j} \in \mathcal{F}}\left|\ln \left\|C_{i}\left(f_{i} \ldots f_{1} ; x, y\right)\right\|\right| .
$$

The system is said to have bounded distortion if $D(n)$ is uniformly bounded.

In Corollary 4.4 below we will show that for compact subsets of $\mathbb{K}_{\alpha, C}^{1}$, bounded distortion is a consequence of semiconformality. To simplify the 
discussion of the relationship between semiconformality and bounded distortion, as well as the estimates that follow in this section and the next, we introduce the following notation:

Definition 4.2. Suppose $\mathcal{F} \subset \mathbb{K}^{1}$. Then we define

$$
Q(n) \equiv \max _{i \leq n} \sup _{f_{i} \in \mathcal{F}, x_{0} \in \bar{I}} \ln \left\|D\left(f_{i} \ldots f_{1}\right)_{\mid x_{0}}\right\| \cdot\left\|\left(D\left(f_{i} \ldots f_{1}\right)_{\mid x_{0}}\right)^{-1}\right\| .
$$

Note that by Definition 1.6, $\mathcal{F}$ is called semiconformal if $Q(n) / n$ tends to zero.

Here is the main estimate of this section. For $X \subset \mathbb{R}^{n},|X|$ denotes its diameter. Also, $S^{n-1}$ denotes the standard unit sphere in $\mathbb{R}^{n}$.

Theorem 4.3. Let $\mathcal{F} \in H\left(\mathbb{K}_{\alpha, C}^{1}\right)$ and let $C_{n}$ and $B_{j}$ be as defined earlier. Then there is a constant $\kappa$ such that for all vectors $v \in S^{n-1}$,

$$
|\ln | C_{n} v|| \leq \kappa \sum_{j=0}^{n-1} e^{Q(j)}\left|B_{j}\right|^{\alpha} .
$$

Proof. For $n \geq 1$ let

$$
\delta_{n}=\left(D f_{n \mid x_{n-1}}\right)^{-1} D f_{n \mid y_{n-1}}-\mathrm{Id} .
$$

Note that because $\mathcal{F}$ and $\bar{I}$ are compact, there is a uniform upper estimate for $\left\|\left(D f_{\mid x}\right)^{-1}\right\|$ on $\mathcal{F} \times \bar{I}$. By the assumption of uniform Hölder continuity it then follows that

$$
\left\|\delta_{i}\right\| \leq \kappa\left|B_{i-1}\right|^{\alpha}
$$

Now

$$
\begin{aligned}
C_{n+1}= & \left(D\left(f_{n} \ldots f_{1}\right)_{\mid x_{0}}\right)^{-1} \cdot\left(\operatorname{Id}+\delta_{n+1}\right) \cdot D\left(f_{n} \ldots f_{1}\right)_{\mid y_{0}} \\
= & \left(D\left(f_{n} \ldots f_{1}\right)_{\mid x_{0}}\right)^{-1} \cdot\left(\operatorname{Id}+\delta_{n+1}\right) \cdot D\left(f_{n} \ldots f_{1}\right)_{\mid x_{0}} \cdot C_{n} \\
= & \left(\operatorname{Id}+\left(D\left(f_{n} \ldots f_{1}\right)_{\mid x_{0}}\right)^{-1} \cdot \delta_{n+1} \cdot D\left(f_{n} \ldots f_{1}\right)_{\mid x_{0}}\right) C_{n} \\
= & \left(\operatorname{Id}+\left(D\left(f_{n} \ldots f_{1}\right)_{\mid x_{0}}\right)^{-1} \cdot \delta_{n+1} \cdot D\left(f_{n} \ldots f_{1}\right)_{\mid x_{0}}\right) \\
& \times\left(\operatorname{Id}+\left(D\left(f_{n-1} \ldots f_{1}\right)_{\mid x_{0}}\right)^{-1} \cdot \delta_{n} \cdot D\left(f_{n-1} \ldots f_{1}\right)_{\mid x_{0}}\right) \ldots\left(\operatorname{Id}+\delta_{1}\right) .
\end{aligned}
$$

Therefore, using Schwarz' inequality (twice) and the triangle inequality, we obtain

$$
\left\|C_{n+1}\right\| \leq \prod_{j=0}^{n}\left(1+\left\|\left(D\left(f_{j} \ldots f_{1}\right)_{\mid x_{0}}\right)^{-1}\right\| \cdot\left\|\delta_{j+1}\right\| \cdot\left\|D\left(f_{j} \ldots f_{1}\right)_{\mid x_{0}}\right\|\right) .
$$

Note that the matrices $C_{n}$ are invertible and that

$$
\left(C_{n}\left(f_{n} \ldots f_{1} ; x_{0}, y_{0}\right)\right)^{-1}=C_{n}\left(f_{n} \ldots f_{1} ; y_{0}, x_{0}\right) .
$$


Thus $\left\|C_{n+1}^{-1}\right\|$ also satisfies equation (4.4). Since

$$
\left\|C_{n}^{-1}\right\|^{-1} \leq\left|C_{n} v\right| \leq\left\|C_{n}\right\|
$$

we now obtain the estimate for $|\ln | C_{n} v||$ upon taking logarithms.

Corollary 4.4. If $\mathcal{F} \in H\left(\mathbb{K}_{\alpha, C}^{1}\right)$ and $\mathcal{F}$ is semiconformal, then $\mathcal{F}$ has bounded distortion.

Proof. From Definition 2.4 it follows that there is a uniform contraction rate $L<1$. Choose $k>0$ such that $e^{-k}=L$. By Theorem 4.3,

$$
D(n) \leq \kappa \cdot 2^{\alpha} \cdot \sum_{j=0}^{n-1} e^{j(Q(j) / j-k \alpha)} \leq A+\sum_{j=N}^{\infty} e^{-j \tau}=A+\frac{e^{-N \tau}}{1-e^{-\tau}}
$$

for suitable positive constants $\kappa, A, \tau$ and $N$.

In the remainder of this section, we discuss the relation between derivatives and sizes of domains. In particular, we apply our distortion result to obtain an estimate, in terms of $D(n)$ and $Q(n)$, of the diameters of images of balls under $\mathcal{F}$. We begin with two simple consequences of the definitions of $D(n)$ and $Q(n)$ :

Proposition 4.5. Let $\mathcal{F} \in H\left(\mathbb{K}^{1}\right)$. Then for all $x_{0}$ and $y_{0}$ in $\bar{I}$ and each unit vector $v$ there is a unit vector $w(v)$ such that

$$
\left|\ln \frac{\left|D\left(f_{n} \ldots f_{1}\right)_{\mid x_{0}}(w(v))\right|}{\left|D\left(f_{n} \ldots f_{1}\right)_{\mid y_{0}}(v)\right|}\right| \leq D(n) .
$$

Proof. Note that

$$
D\left(f_{n} \ldots f_{1}\right)_{\mid x_{0}}\left(C_{n} v\right)=D\left(f_{n} \ldots f_{1}\right)_{\mid y_{0}}(v) .
$$

We choose $w(v)=C_{n} v /\left|C_{n} v\right|$. Then

$$
\frac{\left|D\left(f_{n} \ldots f_{1}\right)_{\mid x_{0}}(w(v))\right|}{\left|D\left(f_{n} \ldots f_{1}\right)_{\mid y_{0}}(v)\right|}=\frac{1}{\left|C_{n} v\right|} .
$$

Now take logarithms and apply Definition 4.1.

The following proposition improves on Proposition 4.5 in that it shows that, up to distortion and deviation from conformality, the asymptotic rate of contraction is independent of the direction in the tangent space as well as initial point.

Proposition 4.6. Let $\mathcal{F} \in H\left(\mathbb{K}^{1}\right)$. Then for all points $x_{0}, y_{0} \in \bar{I}$ and for all unit vectors $u$ at $x_{0}$ and $v$ at $y_{0}$,

$$
\left|\ln \frac{\left|D\left(f_{n} \ldots f_{1}\right)_{\mid x_{0}}(u)\right|}{\left|D\left(f_{n} \ldots f_{1}\right)_{\mid y_{0}}(v)\right|}\right| \leq D(n)+Q(n) .
$$


Proof. We write the left hand side as

$$
\left|\ln \frac{\left|D\left(f_{n} \ldots f_{1}\right)_{\mid x_{0}}(w(v))\right|}{\left|D\left(f_{n} \ldots f_{1}\right)_{\mid y_{0}}(v)\right|}\right|+\left|\ln \frac{\left|D\left(f_{n} \ldots f_{1}\right)_{\mid x_{0}}(u)\right|}{\left|D\left(f_{n} \ldots f_{1}\right)_{\mid x_{0}}(w(v))\right|}\right| .
$$

To the first term we apply the previous result. The second is calculated with the help of

$$
\left\|\left(D\left(f_{n} \ldots f_{1}\right)_{\mid x_{0}}\right)^{-1}\right\|^{-1}|u| \leq\left|D\left(f_{n} \ldots f_{1}\right)_{\mid x_{0}}(u)\right| \leq\left\|D\left(f_{n} \ldots f_{1}\right)_{\mid x_{0}}\right\| \cdot|u|,
$$

and the definition of $Q(n)$.

We now begin our discussion of the effect of $\mathcal{F}$ on the diameters of balls.

Lemma 4.7. Let $A$ and $B$ be connected compact sets in $\mathbb{R}^{n}$ and suppose in addition that $A$ is convex. Suppose that $g: A \rightarrow B$ is a diffeomorphism. Then there are a point $a_{+} \in A$ and a vector $v_{a_{+}} \in T_{a_{+}} A$ (the tangent space to $A$ at $a_{+}$) such that

$$
\frac{\left|D g_{\mid a_{+}}\left(v_{a_{+}}\right)\right|}{\left|v_{a_{+}}\right|} \geq \frac{|B|}{|A|} .
$$

Proof. Let $w$ and $z$ in $B$ be such that $|w-z|=|B|$ and let $x=g^{-1}(w)$ and $y=g^{-1}(z)$. Connect $x$ and $y$ by a straight line segment $\gamma \subset A$ (by the convexity of $A$ ) and parameterize this curve by arc length $(|D \gamma|=1)$. Then

$$
|B|=\left|\int_{0}^{|x-y|} D g(\gamma(t)) \cdot D \gamma(t) d t\right| \leq|A| \max _{x \in A}\left\|D g_{\mid x}\right\| .
$$

Now choose $a_{+}$to be the point where the maximum is assumed.

Lemma 4.8. Let $A$ be a closed ball and $B$ be a set in $\mathbb{R}^{n}$. Suppose that $g: A \rightarrow B$ is a diffeomorphism. Then there is a point $a_{-} \in A$ and $a$ $v_{a_{-}} \in T_{a_{-}} A$ such that

$$
\frac{\left|D g_{\mid a_{-}}\left(v_{a_{-}}\right)\right|}{\left|v_{a_{-}}\right|} \leq \frac{|B|}{|A|} .
$$

Proof. From elementary calculus, we know that

$$
\int_{A} \frac{\left|\operatorname{det} D g_{\mid x}\right|}{\operatorname{vol}(A)} d^{n} x=\frac{\operatorname{vol}(B)}{\operatorname{vol}(A)} \text {. }
$$

The right hand side of this equation is the average of the positive function $\left|\operatorname{det} D g_{\mid x}\right|$. Thus there is an $a_{-} \in A$ such that

$$
\left|\operatorname{det} D g_{\mid a_{-}}\right| \leq \frac{\operatorname{vol}(B)}{\operatorname{vol}(A)} .
$$

Denote the eigenvalues of $D g_{\mid a_{-}}$by $\left\{\lambda_{i}\right\}_{i=1}^{n}$ (counting multiplicity). Observe 
that $\operatorname{vol}(B)$ is no greater than the volume of a ball with diameter $|B|$. Thus

$$
\prod_{i=1}^{n}\left|\lambda_{i}\right| \leq \frac{|B|^{n}}{|A|^{n}}
$$

By taking logarithms and dividing by $n$, it becomes obvious that the average of $\left\{\ln \left|\lambda_{i}\right|\right\}_{i=1}^{n}$ is no greater than $\ln (|B| /|A|)$. Thus there must be an eigenspace $V_{a_{-}}$of $D g_{\mid a_{-}}$satisfying the lemma.

These two lemmas imply the higher dimensional version of the mean value theorem that we will use in the next section.

Corollary 4.9. Let $A$ be a closed ball and $B$ a set in $\mathbb{R}^{n}$. Suppose that $g: A \rightarrow B$ is a diffeomorphism. Then there is a point $a \in A$ and $a v \in T_{a} A$ such that

$$
\frac{\left|D g_{\mid a}(v)\right|}{|v|}=\frac{|B|}{|A|}
$$

Proof. Note that the transformation $T: T A \rightarrow \mathbb{R}^{+}$defined by

$$
T(x, v)=\frac{\left|D g_{\mid x}(v)\right|}{|v|}
$$

is continuous and $T A$ is path-connected. The result is thus a consequence of the previous lemmas.

We now use these results to derive a general statement about scaling in contracting maps.

Theorem 4.10. Let $\mathcal{F} \in H\left(\mathbb{K}^{1}\right)$ and suppose that $f_{a}$ and $f_{b}$ are compositions of at most $n$ functions of $\mathcal{F}$. Then for any ball $B, x \in B$, and $v \in T_{x} B$ we have:

$$
\begin{aligned}
& \left|\ln \left(\frac{\left|f_{a} f_{b}(B)\right|}{\left|f_{a}(B)\right|} \cdot \frac{|B|}{\left|f_{b}(B)\right|}\right)\right| \leq 2 Q(n)+2 D(n), \\
& \left|\ln \frac{\left|D f_{a \mid x}(v)\right|}{|v|}\right| \leq\left|\ln \frac{\left|f_{a}(B)\right|}{|B|}\right|+Q(n)+D(n) .
\end{aligned}
$$

Proof. We first prove (i). The left hand side can be written as

$$
\left|\ln \left(\frac{\left|f_{a} f_{b}(B)\right|}{|B|} \cdot \frac{|B|}{\left|f_{a}(B)\right|} \cdot \frac{|B|}{\left|f_{b}(B)\right|}\right)\right| .
$$

With the help of Corollary 4.9, we get

$$
\frac{\left|f_{a} f_{b}(B)\right|}{|B|}=\frac{\left|\left(D f_{a} \cdot D f_{b}\right)_{\mid x}\left(v_{x}\right)\right|}{\left|v_{x}\right|}=\frac{\left|D f_{a \mid y}\left(v_{y}\right)\right|}{\left|v_{y}\right|} \cdot \frac{\left|D f_{b \mid x}\left(v_{x}\right)\right|}{\left|v_{x}\right|}
$$

where $v_{y}$ is a unit vector in the direction of $D f_{b \mid x}\left(v_{x}\right)$. The other derivatives 
can also be calculated with the help of the same corollary, to give

$$
\frac{\left|f_{a} f_{b}(B)\right|}{\left|f_{a}(B)\right|} \cdot \frac{|B|}{\left|f_{b}(B)\right|}=\frac{\left|D f_{a \mid x_{1}}\left(v_{1}\right)\right|}{\left|D f_{a \mid x_{2}}\left(v_{2}\right)\right|} \cdot \frac{\left|D f_{b \mid y_{1}}\left(w_{1}\right)\right|}{\left|D f_{b \mid y_{2}}\left(w_{2}\right)\right|} .
$$

The result now follows from Proposition 4.6.

Now we prove (ii):

$$
\ln \frac{\left|D f_{a \mid x}(v)\right|}{|v|}=\ln \frac{\left|D f_{a \mid q}\left(v_{0}\right)\right|}{\left|v_{0}\right|}+\ln \frac{\left|D f_{a \mid x}(v)\right|}{\left|D f_{a \mid q}\left(v_{0}\right)\right|},
$$

where $q$ and $v_{0}$ are chosen such that Corollary 4.9 applies and the second term of the left hand side is evaluated with the help of Proposition 4.6.

5. The dimension estimate. We prove that if $\mathcal{F} \in H\left(\mathbb{K}_{\alpha, C}^{1}\right)$ is a semiconformal differentiable iterated function system, then it is a point of lower semicontinuity of the function that evaluates the Hausdorff dimension. We note here that if $\mathcal{F}$ is a one-dimensional system with finitely many branches and satisfying a strong condition on the distance of the individual branches, then the Hausdorff dimension varies continuously (see [24] or [20] for more information). Without that condition, it is clear that the dimension is not continuous as observed in the introduction. Nonetheless, the proof of the semicontinuity given here has some resemblance to Takens' proof.

For a given system $\mathcal{F} \in H\left(\mathbb{K}^{1}\right)$, we choose positive constants $K=K(\mathcal{F})$ and $k=k(\mathcal{F})$ such that for all $f \in \mathcal{F}$ and $x \in \bar{I}$,

$$
\left(\left\|\left(D f_{\mid x}\right)^{-1}\right\|\right)^{-1}>e^{-K}, \quad\left\|D f_{\mid x}\right\|<e^{-k} .
$$

Note that by continuity (5.1) automatically holds for all $\mathcal{F}^{\prime}$ in a $\mathbb{K}^{1}$-neighborhood of $\mathcal{F}$ (see Definition 2.3).

A dynamic cover $\mathcal{U}$ of $\Lambda(\mathcal{F})$ is a finite cover by open sets each of which can be written as $f_{1} \ldots f_{n}(I), f_{i} \in \mathcal{F}$.

Lemma 5.1. Suppose $\mathcal{F} \in \mathbb{K}^{1}$. Then for each $n>0$, there is a dynamic cover $\mathcal{U}_{n}$ of $\Lambda(\mathcal{F})$ such that for all $U \in \mathcal{U}_{n}$,

$$
2 e^{-2 Q(n)-2 D(n)-n k-K} \leq|U|<2 e^{-n k} .
$$

Furthermore, all elements of $\mathcal{U}_{n}$ are of the form $f_{m} \ldots f_{1}(I)$ with $m \leq n$.

Proof. Consider a sequence $\alpha=\left\{f_{i}\right\}_{i=1}^{\infty}$ of elements of $\mathcal{F}$. For each $n>0$ let $g_{\alpha, n}=f_{1} \circ \ldots \circ f_{i_{n}}$, where $n$ is the smallest positive integer for which

$$
\left|g_{\alpha, n}(I)\right|=\left|f_{1} \circ \ldots \circ f_{i_{n}}(I)\right|<2 e^{-n k} \text {. }
$$

We claim that then also

$$
2 e^{-2 Q(n)-2 D(n)-n k-K} \leq\left|g_{\alpha, n}(I)\right| .
$$

Certainly this is true when $n=1$, for $i_{1}=1$, and

$$
2 e^{-K} \leq\left|f_{1}(I)\right|<2 e^{-k}
$$


by (5.1) and Corollary 4.9. We continue by induction. Suppose (5.2) holds for $n=m$. Note that it follows from (5.1) that either $i_{m+1}=i_{m}$ (in which case (5.2) follows immediately for $n=m+1$ ) or $i_{m+1}=i_{m}+1$. In the latter case we have

$$
2 e^{-(m+1) k} \leq\left|g_{\alpha, m}(I)\right|<2 e^{-m k} .
$$

Also, as a consequence of (5.1) and Theorem 4.10 we then have

$$
\left|g_{\alpha, m+1}(I)\right|=\left|g_{\alpha, m} \circ f_{i_{m+1}}(I)\right|=C_{m} \frac{\left|f_{i_{m+1}}(I)\right|}{|I|}\left|g_{\alpha, m}(I)\right|,
$$

where

$$
C_{m} \geq e^{-2 Q(m)-2 D(m)} \quad \text { and } \quad e^{-K} \leq \frac{\left|f_{i_{m+1}}(I)\right|}{|I|}<e^{-k} .
$$

Combining this with (5.3) we get

$$
e^{-2 Q(m)-2 D(m)-(m+1) k-K} \leq\left|g_{\alpha, m+1}(I)\right|,
$$

which implies (5.2) and so completes the induction.

Now we let $\mathcal{U}_{n}=\left\{g_{\alpha, n}(I): \alpha\right.$ a sequence in $\left.\mathcal{F}\right\}$. Then $\mathcal{U}_{n}$ is a covering of $\Lambda(\mathcal{F})$ because for each $x \in \Lambda(\mathcal{F})$ there is an infinite sequence $\alpha=\left\{f_{i}\right\}_{i=1}^{\infty}$ such that

$$
x=\bigcap_{n>0} f_{1} \circ \ldots \circ f_{n}(\bar{I}) .
$$

Since $f_{n}(\bar{I}) \subset I$ it follows that we also have

$$
x=\bigcap_{n>0} f_{1} \circ \ldots \circ f_{n}(I) .
$$

In particular, $x \in g_{\alpha, n}(I) \in \mathcal{U}_{n}$. The result follows from the compactness of $\Lambda(\mathcal{F})$.

For each $n$, let $\mathcal{F}_{n}$ denote the compositions of elements of $\mathcal{F}$ that define the dynamic cover $\mathcal{U}_{n}$. That is,

$$
f=f_{1} \circ \ldots \circ f_{m} \in \mathcal{F}_{n} \Leftrightarrow f(I) \in \mathcal{U}_{n} .
$$

With each dynamic cover $\mathcal{U}_{n}=\mathcal{F}_{n}(I)$ of $\Lambda(\mathcal{F})$ we associate a compact subset $\Lambda_{n}$ of $\Lambda$ in the following way: Let $\mathcal{V}_{n}$ be a maximal collection of disjoint members of $\mathcal{U}_{n}$. Thus by construction each $V \in \mathcal{V}_{n}$ is of the form $f(I)$ where $f$ belongs to a subset $\mathcal{G}_{n}$ of $\mathcal{F}_{n}$. Clearly, $\mathcal{G}_{n}$ is a differentiable iterated function system consisting of a finite number, say $N_{n}$, of diffeomorphisms, and its invariant set $\Lambda_{n}=\Lambda\left(\mathcal{G}_{n}\right)$ is a subset of $\Lambda$. The main reason for introducing the systems $\mathcal{G}_{n}$ is, of course, that they satisfy the strong separation condition (Definition 1.7). Therefore, the Hausdorff dimensions of their invariant sets are easy to calculate. These dimensions serve as approximations to the dimension of $\Lambda$ (see Proposition 5.4). 
Lemma 5.2. Suppose $\mathcal{F} \in \mathbb{S}$. Let $\mathcal{G}_{n}$ be the differentiable iterated function system constructed as above, and suppose it consists of $N_{n}$ diffeomorphisms. Then

$$
\operatorname{Hdim}\left(\Lambda_{n}\right) \leq \operatorname{Hdim}(\Lambda) \leq \limsup _{n \rightarrow \infty} \frac{\ln N_{n}}{n k} .
$$

Proof. The first of the inequalities is obvious because of the inclusion $\Lambda_{n} \subseteq \Lambda$. For the second inequality let $d_{n}=\left(\ln N_{n}\right) /(n k)$ and let $d=$ $\lim \sup _{n \rightarrow \infty} d_{n}$. If $d=\infty$, the second inequality is obvious, so we assume $d<\infty$.

For each $V$ in $\mathcal{V}_{n}$, choose $x_{V} \in V$ and let $\widetilde{V}$ be the ball with center $x_{V}$ but with radius $4 e^{-n k}$. Note that the collection $\widetilde{\mathcal{V}}_{n}$ of these sets covers $\Lambda$. Then $N_{n}=e^{n k d_{n}}$ and, for any $\varepsilon>0$,

$$
\mathcal{H}_{8 e^{-n k}}^{\varepsilon+d_{n}}(\Lambda) \leq \sum_{\widetilde{V} \in \tilde{\mathcal{V}}_{n}}|\widetilde{V}|^{\varepsilon+d_{n}}=N_{n}\left(8 e^{-n k}\right)^{\varepsilon+d_{n}}=8^{\varepsilon+d_{n}} e^{-n k \varepsilon} .
$$

Therefore $\mathcal{H}_{8 e^{-n k}}^{\varepsilon+d_{n}}(\Lambda)$ tends to zero as $n$ goes to infinity. Because

$$
\mathcal{H}_{8 e^{-n k}}^{2 \varepsilon+d}(\Lambda) \leq \mathcal{H}_{8 e^{-n k}}^{\varepsilon+d_{n}}(\Lambda)
$$

for large $n$, this establishes the upper bound for the Hausdorff dimension.

The actual calculation of the Hausdorff dimension uses the following result.

Proposition 5.3. Let the system $\mathcal{H} \in \mathbb{K}^{1}$ be a set of $N$ contractions satisfying the strong separation condition. Suppose further that

$$
0<e^{\lambda_{-}} \leq \frac{\left|D h_{\mid x}(v)\right|}{|v|} \leq e^{\lambda_{+}}<1
$$

for all $h \in \mathcal{H}, x \in \bar{I}$ and $v \in T_{x} \bar{I}$. Then

$$
\frac{-\ln N}{\lambda_{-}} \leq \operatorname{Hdim}(\Lambda(\mathcal{H})) \leq \frac{-\ln N}{\lambda_{+}} .
$$

This is found as Theorem 8.8 in [2].

Proposition 5.4. Consider the invariant sets $\Lambda_{n}$ of the systems $\mathcal{G}_{n}$ derived from the system $\mathcal{F} \in \mathbb{S}$ and consisting of $N_{n}$ contractions satisfying the strong separation condition. Let $k$ be the constant defined in (5.1). Then

$$
\lim _{n \rightarrow \infty}\left|\operatorname{Hdim}\left(\Lambda_{n}\right)-\frac{\ln N_{n}}{n k}\right|=0
$$

Proof. The proof consists of estimating the numbers $\lambda_{-}$and $\lambda_{+}$of the previous proposition for the systems $\mathcal{G}_{n}$. Let $g: I \rightarrow V \in \mathcal{V}_{n}$ be a member of the finite family $\mathcal{G}_{n}$. By construction, the map $g$ is a composition of $m \leq n$ diffeomorphisms $f \in \mathcal{F}$. So for any $x \in \bar{I}$ and $v$ in the tangent space $T_{x} \bar{I}$, 
Lemma 5.1 and Theorem 4.10(ii) give

$$
-n k-K-3 D(n)-3 Q(n) \leq \ln \frac{\left|D g_{\mid x}(v)\right|}{|v|} \leq-n k+D(n)+Q(n) .
$$

Thus, by Proposition 5.3,

$$
\frac{\ln N_{n}}{n k+K+3 D(n)+3 Q(n)} \leq \operatorname{Hdim}\left(\Lambda\left(\mathcal{G}_{n}\right)\right) \leq \frac{\ln N_{n}}{n k-D(n)-Q(n)} .
$$

By semiconformality, as $n \rightarrow \infty$, this establishes the result.

Recall the definition of the limit capacity, given in Section 1. The limit capacity is always at least as big as the Hausdorff dimension, because for the former we insist that the covering sets all have the same diameter. The following is an immediate consequence of Proposition 5.4 and Lemma 5.2:

Corollary C. Suppose that $\mathcal{F} \in \mathbb{S}$. Then the limit capacity of $\Lambda(\mathcal{F})$ is equal to its Hausdorff dimension.

Everything is now in place to prove the continuity part of Theorem B. The methods are exactly the same as those used in the previous proposition.

THEOREM 5.5. Every point of $\mathbb{O} \cap \mathbb{S}$ is a point of continuity of the function Hdim on $\mathbb{O}$.

Proof. For $\mathcal{F} \in \mathbb{O} \cap \mathbb{S}$ and $\mathcal{F}^{\prime} \in \mathbb{O}$ we let $\mathcal{F}_{n}$ and $\mathcal{F}_{n}^{\prime}$ be the collections of iterates associated with the dynamic covers of $\Lambda(\mathcal{F})$ and $\Lambda\left(\mathcal{F}^{\prime}\right)$ respectively. If $\mathcal{F}$ and $\mathcal{F}^{\prime}$ are sufficiently close in the metric $\operatorname{Hd}_{1}$, then they will satisfy (5.1) for the same constants $k$ and $K$. Because $\mathcal{F}, \mathcal{F}^{\prime} \in \mathbb{O}$ we have $\Lambda\left(\mathcal{F}_{n}\right)=$ $\Lambda(\mathcal{F})$ and $\Lambda\left(\mathcal{F}_{n}^{\prime}\right)=\Lambda\left(\mathcal{F}^{\prime}\right)$. We let $N_{n}=\#\left(\mathcal{F}_{n}\right)$, and we define $D(n)$ and $Q(n)$ for $\mathcal{F}$ as in Definitions 4.1 and 4.2. For $\mathcal{F}^{\prime}$ we denote the corresponding quantities by $D^{\prime}(n)$ and $Q^{\prime}(n)$. Then by (5.4) in the proof of Proposition 5.4,

$$
\frac{\ln N_{n}}{n k+K+3 D(n)+3 Q(n)} \leq \operatorname{Hdim}(\Lambda(\mathcal{F})) \leq \frac{\ln N_{n}}{n k-D(n)-Q(n)}
$$

for each $n$. A similar inequality holds for $\operatorname{Hdim}\left(\Lambda\left(\mathcal{F}^{\prime}\right)\right)$.

Fix $n$. Then for $\mathcal{F}^{\prime}$ in a sufficiently small neighborhood $\mathcal{N}$ of $\mathcal{F}$ in $\mathbb{O}$ it follows that $\#\left(\mathcal{F}_{n}^{\prime}\right)=N_{n}$, and that $D^{\prime}(n)$ and $Q^{\prime}(n)$ are arbitrarily close to $D(n)$ and $Q(n)$ respectively. Thus, by choosing $n$ large initially, and $\mathcal{N}$ small, we can make $\left|\operatorname{Hdim}(\Lambda(\mathcal{F}))-\operatorname{Hdim}\left(\Lambda\left(\mathcal{F}^{\prime}\right)\right)\right|$ as small as desired.

Here is the remaining half of Theorem B.

THEOREM 5.6. Every point of $\mathbb{S}$ is a point of lower semicontinuity of the function Hdim on $\mathbb{K}^{1}$.

Proof. Recall that $\Lambda_{n}=\Lambda_{n}(\mathcal{F})$ is the invariant set of the system $\mathcal{G}_{n}=$ $\mathcal{G}_{n}(\mathcal{F})$ satisfying the strong separation condition. For a given $\mathcal{F} \in \mathbb{S}$ choose $n$ so that

$$
\left|\operatorname{Hdim}(\Lambda(\mathcal{F}))-\operatorname{Hdim}\left(\Lambda_{n}(\mathcal{F})\right)\right|<\varepsilon / 2 .
$$


This is possible by Proposition 5.4. If we choose $\mathcal{F}^{\prime} \in \mathbb{K}^{1}$ sufficiently close to $\mathcal{F}$, then for each composition $f_{1} \circ \ldots \circ f_{n} \in \mathcal{G}_{n}$ we can choose a composition $f_{1}^{\prime} \circ \ldots \circ f_{n}^{\prime}$ with $f_{i}^{\prime} \in \mathcal{F}^{\prime}$ close to $f_{i}$. Let $\mathcal{G}_{n}^{\prime}$ denote the collection of these compositions.

Then $\mathcal{G}_{n}^{\prime}$ also satisfies the strong separation condition. We assume, using Theorem 5.5, that

$$
\left|\operatorname{Hdim}\left(\Lambda_{n}\left(\mathcal{F}^{\prime}\right)\right)-\operatorname{Hdim}\left(\Lambda_{n}(\mathcal{F})\right)\right|<\varepsilon / 2 .
$$

Then, by Lemma 5.2,

$\operatorname{Hdim}\left(\Lambda\left(\mathcal{F}^{\prime}\right)\right) \geq \operatorname{Hdim}\left(\Lambda_{n}\left(\mathcal{F}^{\prime}\right)\right) \geq \operatorname{Hdim}\left(\Lambda_{n}(\mathcal{F})\right)-\varepsilon / 2 \geq \operatorname{Hdim}(\Lambda(\mathcal{F}))-\varepsilon$

\section{References}

[1] C. J. Bishop and P. W. Jones, Hausdorff dimension and Kleinian groups, Acta Math. 179 (1997), 1-39.

[2] K. J. Falconer, The Geometry of Fractal Sets, Cambridge Univ. Press, 1985.

[3] -, Dimensions and measures of quasi self-similar sets, Proc. Amer. Math. Soc. 106 (1989), 543-554.

[4] -, Fractal Geometry. Mathematical Foundations and Applications, Wiley, 1990.

[5] -, Bounded distortion and dimension for nonconformal repellers, Math. Proc. Cambridge Philos. Soc. 115 (1994), 315-334.

[6] J. Graczyk, L. Jonker, G. Świątek, F. M. Tangerman and J. J. P. Veerman, Differentiable circle maps with a flat interval, Comm. Math. Phys. 173 (1995), 599-622.

[7] K. Gröchenig and A. Haas, Self-similar lattice tilings, J. Fourier Anal. Appl. 1 (1994), 131-170.

[8] J. E. Hutchinson, Fractals and self-similarity, Indiana Univ. Math. J. 30 (1981), 713-747.

[9] Y. Jiang, Dynamics of certain non-conformal semigroups, Complex Variables 22 (1993), 27-34.

[10] R. Kenyon, Projecting the one dimensional Sierpinski gasket, Israel J. Math. 94 (1997), 221-238.

[11] J. C. Lagarias and Y. Wang, Integral self-affine tilings in $\mathbb{R}^{n} I$, J. London Math. Soc. (2) 54 (1996), 161-179.

[12] -, - Tiling the line with translates of one tile, Invent. Math. 124 (1996), 341-365.

[13] J. Marstrand, Some fundamental geometrical properties of plane sets of fractional dimension, Proc. London Math. Soc. 4 (1954), 257-302.

[14] H. McCluskey and A. Manning, Hausdorff dimension of horseshoes, Ergodic Theory Dynam. Systems 3 (1983), 251-260.

[15] W. de Melo and S. van Strien, One Dimensional Dynamics, Springer, 1993.

[16] J. Palis and M. Viana, On the continuity of the Hausdorff dimension, in: Lecture Notes in Math. 1331, Springer, 1988, 150-161.

[17] M. Pollicott and K. Simon, The Hausdorff dimension of $\lambda$-expansions with deleted digits, Trans. Amer. Math. Soc. 347 (1995), 967-983.

[18] P. Raith, Continuity of the Hausdorff dimension for piecewise monotonic maps, Israel J. Math. 80 (1992), 97-133. 
[19] D. Ruelle, Repellers for real analytic maps, Ergodic Theory Dynam. Systems 2 (1982), 99-107.

[20] - Bowen's formula for the Hausdorff dimension of self-similar sets, in: Scaling and Self-Similarity in Physics, Progr. Phys. 7, Birkhäuser, 1983, 351-359.

[21] T. Sauer, J. A. Yorke and M. Casdagli, Embedology, J. Statist. Phys. 65 (1991), 579-616.

[22] B. Solomyak, On the random series $\sum \pm \lambda^{n}$ (an Erdös problem), Ann. of Math. 142 (1995), 611-625.

[23] G. Świątek and J. J. P. Veerman, On a conjecture of Furstenberg, Israel J. Math., to appear.

[24] F. Takens, Limit capacity and Hausdorff dimension of dynamically defined sets, Lecture Notes in Math. 1331, Springer, 1988, 197-211.

[25] J. J. P. Veerman, Two-dimensional generalizations of Haar bases, in: Internat. Conf. on Dynamical Systems, F. Ledrappier et al. (eds.), Pitman Res. Notes Math. Ser. 362, Longman, 1996, 220-235.

[26] -, Intersecting self-similar Cantor sets, Bol. Soc. Brasil. Mat. 26 (1995), 167-181.

[27] - The dynamics of well-ordered orbits, lecture notes, Univ. of Barcelona, 1995.

[28] J. J. P. Veerman and L. B. Jonker, Rigidity properties of locally scaling fractals, preprint \# 1997/2, Inst. for Math. Soc., SUNY, Stony Brook.

[29] J. J. P. Veerman and B. D. Stošić, On the dimensions of certain incommensurably constructed sets, Experiment. Math. 9 (2000), 413-423.

[30] L. S. Young, Dimension, entropy and Lyapunov exponents, Ergodic Theory Dynam. Systems 2 (1982), 109-124.

Queen's University

Kingston, Ontario, Canada

E-mail: leo@mast.queensu.ca
Department of Mathematical Sciences Portland State University Portland, OR 97207, U.S.A. E-mail: veerman@pdx.edu

Received 6 March 2000;

in revised form 1 August 2001 and 24 January 2002 